\title{
ОТДАЛЕННЫЕ РЕЗУЛЬТАТЫ ХИРУРГИЧЕСКОГО ЛЕЧЕНИЯ ПОСТЬЯЗВЕННОГО СТЕНОЗА ПИЛОРИЧЕСКОГО КАНАЛА
}

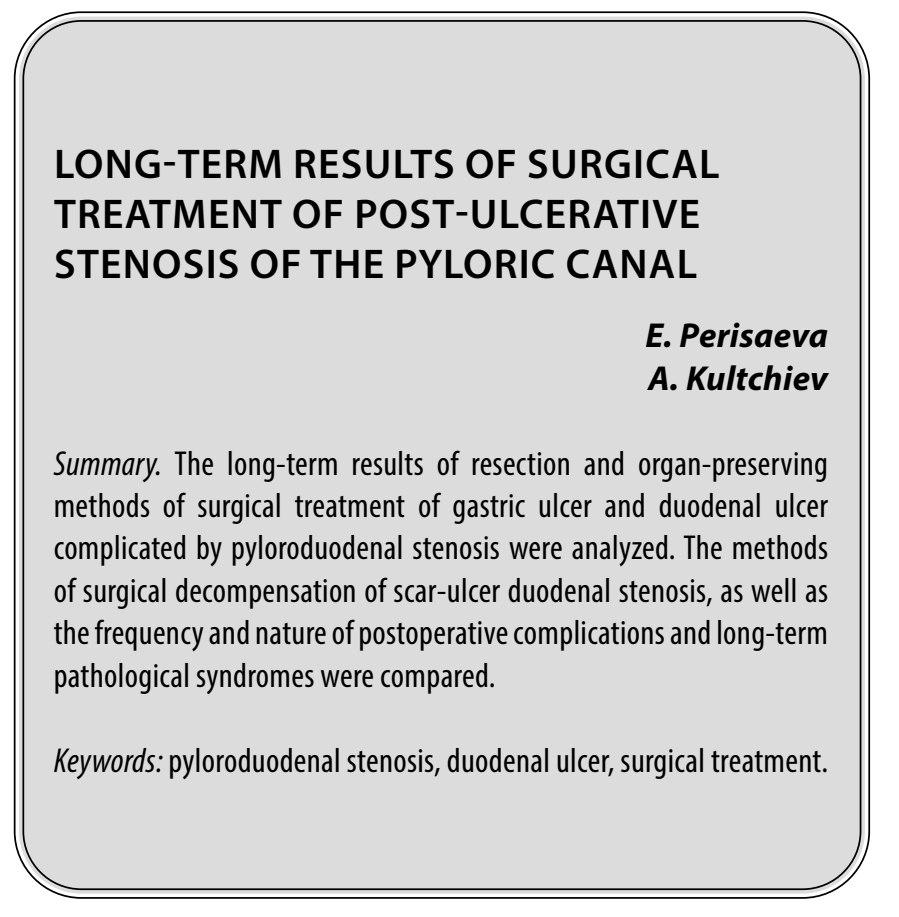

\section{Актуальность проблемы}

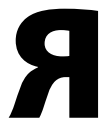

звенная болезнь желудка и двенадцатиперстной кишки является одной из самых распространенных заболеваний желудочно-кишечного тракта среди взрослого населения и нередко ведет к развитию угрожающих жизни осложнений. Одним из таких осложнений является стенозирование выходного отдела желудка и ДПК, который по сводным данным ряда авторов наблюдается в 45-60\% случаев[2,8,10]. Подавляющее большинство этих пациентов нуждается в хирургическом лечении. Поэтому проблема хирургической тактики при различных стадиях стеноза приобретают особую актуальность. К сожалению, вопрос о выборе метода и тактики хирургического лечения не решен до сих пор и остается предметом широкой дискуссии[1,2,3,5,6,7,8,11,12]. Все имеющиеся методы подразделяются на 2 группы: резекционные и органосохраняющие методики. Резекция желудка, которая сопровождается относительно высокими цифрами летальности (2-5\%), значительным числом ранних послеоперационных осложнений (12-35\%) и развитием различных постгастрорезекционных синдромов (25-60\%) в отдаленном периоде, а также повышенным риском возникновения рака культи желудка (4-6\%), по-прежнему занимает лидирующие позиции в хирургическом ле-
Перисаева Элина Акимовна Ассистент, ФГБОУ ВО «Северо-Осетинская государственная медицинская академия»

(2. Владикавказ)

perisaeva.elinka@yandex.ru

Кульчиев Ахсарбек Агубеевич

Д.м.н., профессор, ФГБОУ ВО «Северо-Осетинская государственная медицинская академия»

(2. Владикавказ)

kulchiev.ahsarbek@yandex.ru

Аннотация. Проанализированы отдаленные результаты резекционных и органосохраняющих методов хирургического лечения язвенной болезни желудка и ДПК, осложненной пилородуоденальным стенозом. Проведена сравнительная оценка способов хирургического лечения декомпенсированного рубцово-язвенного стеноза (ДРЯС) ДПК, а так же частота и характер послеоперационных осложнений и отдаленных патологических синдромов.

Ключевые слова: пилородуоденальный стеноз, язвенная болезнь ДПК, хирургическое лечение.

чении язвенной болезни в целом и в особенности ДРЯС ДПК. [4,5]. В течение последних 15-20 лет в лечении осложненных форм ЯБДПК, в том числе и ДРЯС, стали применяться органосохраняющие операции, преимуществами которых являются меньшая травматичность, минимальная летальность, низкая частота развития ранних послеоперационных осложнений и патологических синдромов в отдаленном периоде[2,4,5,9].

\section{Цель исслеАования}

Обоснование выбора хирургической тактики и сравнительная оценка методов хирургического лечения ДРЯС ДПК у данной категории пациентов.

\section{Материалы и метолы}

Для проведения анализа использован обширный опыт хирургического лечения стенозов (резекций желудка более 1000 операций, различные варианты и органосохраняющих операций с ваготомией- 860) ДПК язвенного генеза дает нам основание отдавать предпочтение дренирующим желудок операциям с СПВ. Кроме того, при наличии ограниченного ряда показаний, у отдельной категории больных, считаем патогенетически обоснованным антрумэктомию со стволовой ва- 
готомией и гастроэнтероанастомоз по Ру - Агеенко. При язвенной болезни желудка и ДПК одним из основных патогенетических условий операции считается сохранение пассажа по ДПК. Однако, в исключительных случаях (сочетание язвенной болезни с выраженными формами функциональной ХДН), вынуждены выключать из пассажа ДПК.

На первом этапе, до 1985 г., по поводу различных форм язвенного стеноза ДПК оперировано 86 больных - І группа.

На втором этапе, с 1986 по 2018 г. с диагнозом хроническая язвенная болезнь осложненная стенозом выполнено 126 операций - II группа.

У второй группы больных количество резекционных вмешательств резко сократилось - до 6. Абсолютное большинство операций составило органосохраняющие с СПВ - 120 (95,2\%) из 126 больных в этой группе больных нами выявлено всего 6 резекций желудка, в объеме - антрумэктомия со стволовой ваготомией. Это те случаи, когда нельзя было сохранить пассаж по ДПК - функциональная дуоденальная непроходимость субкомпенсированной и декомпенсированной стадии.

Из дренирующих желудок операций предпочтение отдаем гастродуоденостомии по Финнею, с учетом имеющейся возможности иссечения краев язвы и рубцовой ткани в пределах здоровых тканей, при поражении передней и боковых стенок ДПК, а так же ревизии задней стенки ДПК.

Следует отметить, что процент общих послеоперационных осложнений в группе с органосохраняющими операциями, в два раза меньше по сравнению с резекционными, что подтверждает патогенетическую обоснованность объема выполненных операций.

Такие грозные осложнения, как несостоятельность культи ДПК, анастомоза и послеоперационный панкреатит чаще наблюдали в I группе, после резекции желудка. В этой группе имели место два случая несостоятельности культи ДПК и один - несостоятельность анастомоза после Бильрот-І с одним летальным исходом. Еще один больной погиб от послеоперационного панкреатита, развившегося вследствие попытки закрытия трудной культи с пенетрирующей язвой в головку поджелудочной железы. В I группе имелось 2 (2,3\%) летальных случая.

Bo II группе подобных тяжелых послеоперационных осложнений не было. Наиболее частым послеоперационным осложнением в этой группе было нагноение раны, что никак нельзя объяснить с характером объема операции. Характерные для II группы операции осложнения - гастростаз и диарея имели место в единичных случаях и легко купировались медикаментозно.

В I группе больных выбор объема операции не зависел от степени стеноза. В этой группе больных выбор метода резекции в большей степени зависел от надежности культи ДПК и гастродуоденоанастомоза. При оптимальной ситуации пытались выполнить резекцию по Бильрот -І в 12 (14\%) случаях. При закрытии «трудной» культи ДПК - гастроэнтероанастомоз по Ру - 21 $(24,4 \%)$ больной. У абсолютного большинства выполнена резекция по Гофмейстеру - Финстереру - 53 $(61,6 \%)$ пациента.

Bo II группе больных степень выраженности стеноза влияла на выбор метода дренирующей желудок операции. При IV степени стеноза, при резком угнетении перистальтических возможностей стенки желудка, оптимальным считаем широкое соустье между желудком и ДПК по Финнею. Эту методику считаем оптимальной и при сочетании стеноза с пенетрацией язвы в головку поджелудочной железы. При II и III степени стеноза, когда можно сохранить пилорический жом, предпочтение отдавали дуоденопластике с обязательной СПВ. Некоторые ведущие хирурги выполняют дуоденопластику без СПВ. Мы считаем обязательным СПВ в плановой хирургии, при всех органосохраняющих операциях язвенной болезни ДПК.

В этой группе (II) было выполнено 6 антрумэктомий с стволовой ваготомией по «вынужденным» показаниям - ХДН III - IV ст.

Таким образом, у абсолютного большинства больных во II группе выполнена операция Финнея с СПВ (72,3\%). Пилоропластика по Гейнеке - Микуличу выполнена у 8 (6,3\%) больных при II степени стеноза.

В табл. 1 (I группе) отражены функциональные и органические изменения выявленных в отдаленном периоде после резекционных операций. Как видно из данной таблицы, абсолютное большинство (67,2\%) пациентов, после различных, резекционных желудок операций, имеют различные патологические состояния. Из них - у 7,8\% рецидив язвенной болезни. Из резекционных желудок методов операций наихудшие результаты получены после операции Гофмейстера Финстерера. У 97,5\% пациентов отмечены разные патологические состояния.

В таблице 1 особо следует обратить внимание на тот факт, что после резекции желудка, по Гофмейстеру Финстереру в отдаленном периоде нет пациентов, которые чувствуют себя абсолютно здоровыми. 
Таблица 1. (I - группа). Отдаленные результаты

\begin{tabular}{|c|c|c|c|c|}
\hline \multirow{2}{*}{ Патологическое состояние } & \multicolumn{3}{|c|}{ Результат операции } & \multirow{2}{*}{$\begin{array}{l}\text { Всего } \\
(64)\end{array}$} \\
\hline & Г. $-\Phi .(41)$ & по Рy (14) & $5-I(9)$ & \\
\hline Рецидив язвы & $4(9,7)$ & 1 & - & $5(78 \%)$ \\
\hline Демпинг синдром & $7(17 \%)$ & - & - & $7(17 \%)$ \\
\hline Синдром приводящей петли & 2 & - & - & 2 \\
\hline Желудочный дискомфорт & $12(30 \%)$ & 2 & - & $14(22 \%)$ \\
\hline Вегетоастенический синдром & $10(24 \%)$ & - & 1 & $11(17 \%)$ \\
\hline Железодефицитная анемия & $3(7,3 \%)$ & - & 1 & $4(9,4 \%)$ \\
\hline Итого & $38(97,5 \%)$ & $3(21,4 \%)$ & $2(22,2 \%)$ & \begin{tabular}{|l|}
43 \\
$(67,2 \%)$
\end{tabular} \\
\hline
\end{tabular}

Таблица 2. (II - группа). Отдаленные результаты

\begin{tabular}{|c|c|c|c|c|c|}
\hline \multirow[b]{2}{*}{ Патологическое состояние } & \multicolumn{4}{|l|}{ Операция } & \multirow[b]{2}{*}{ Всего (83) } \\
\hline & $\begin{array}{l}\text { Финнея, с СПВ } \\
-51\end{array}$ & $\begin{array}{l}\text { Гейнеке } \\
\text { Микуличу, СПВ } \\
-6\end{array}$ & \begin{tabular}{|l|} 
Дуоденопласти- \\
ка, СПВ - 21
\end{tabular} & $\begin{array}{l}\text { Антрум } \\
\text { эктомия, СТВ, } \\
\text { Ру. - } 6\end{array}$ & \\
\hline Рецидив язвы & 2 & - & - & - & $2(2,4)$ \\
\hline Демпинг синдром легкой степени & 1 & 1 & - & - & 2 \\
\hline Диарея & 1 & - & - & - & 1 \\
\hline Желудочный дискомфорт & 4 & 1 & 1 & 1 & $7(8,3 \%)$ \\
\hline Рубцовый стеноз анастомоза & - & - & 1 & - & 1 \\
\hline Вегетоастенический синдром & 4 & - & - & 1 & 5 \\
\hline Итого & $12(23,5 \%)$ & 2 & 2 & 2 & $18(21,6) \%$ \\
\hline
\end{tabular}

Bo II группе больных (табл. 2) отдаленные результаты более благоприятны по сравнению с I группой. Как видно из таблицы № 2, разные патологические состояния после органосохраняющих желудок операций с СПВ, выявлено у 21,6\%. По сравнению с I группой - в 3 раза меньше. Из них рецидив язвы в 2,4\%. Наиболее частым патологическим состоянием в этой группе является желудочный дискомфорт у 8,3\%.

Таким образом, наглядно видно, что у абсолютного большинства больных 78,4\% после органосохраняющих желудок операций с СПВ в отдаленном периоде не выявлено патологическое состояние. У 2,4\% имеется рецидив язвенной болезни.

Сегодня результаты любых хирургических операций оцениваются по отдаленным результатам, основываясь на качестве жизни пациента. С этих позиций нами изучены полученные отдаленные результаты выполненных операций в разных группах больных.

\section{Оченка качества жизни больных в отАаленном периоле}

Полученные отдаленные результаты любых операций с позиции доказательной медицины, должны отра- жать качество жизни пациента. С этих позиций нами изучены отдаленные результаты по классификации Visick, опросник и DDQ - 15 в отдаленном периоде, в сроки от 3 до 30 лет.

После резекционных операций (I - группа) в отдаленные сроки качество жизни определено у 64 пациентов и у 83 во II группе.

Мы провели анкетирование всех 147 больных перенесших операции на желудке, по обоим опросникам и классификации Visick.

Согласно проведенным расчетам, среднее значение индекса DDQ - 15 для здоровых лиц составляет - 4,13, ИКЖФХК - 124,1 +5,4 балла, а в группах с «отличными» и «хорошими» результатами операции следует относить с величиной индекса DDQ - 15 более - 3,41, ИКЖФХК - более 100 баллов.

В этой группе пациентов лишь у 27,3\% пациентов отдаленные показатели качества жизни можно признать приемлемыми, в то время как у абсолютного большинства $(72,7 \%)$ качество жизни нельзя признать хорошим. И лишь у $2(3,1 \%)$ пациентов оно признанно отличным. 
Таблица 3. Отдаленные результаты резекционных и органосохраняющих операций в сочетании с ваготомией.

\begin{tabular}{|c|c|c|c|c|}
\hline \multirow{2}{*}{ Результат } & \multicolumn{2}{|c|}{ I - группа (резекция желудка) } & \multicolumn{2}{|c|}{$\begin{array}{l}\text { II - группа (органосохраняющая операция } \\
\text { + ваготомия) }\end{array}$} \\
\hline & A6c. & $\%$ & A6c. & $\%$ \\
\hline Отличный & & & 60 & 72,8 \\
\hline Хороший & 17 & 26,2 & 11 & 12,0 \\
\hline Удовлетворительный & 33 & 52,0 & 9 & 11,6 \\
\hline Плохой & 14 & 21,9 & 3 & 3,6 \\
\hline Итого & 64 & 100 & 83 & 100 \\
\hline
\end{tabular}

Качество жизни по DDQ - 15 у больных перенесших резекции желудка близка по показателям качества ИКЖФХК - хорошее и отличное качество жизни выявлено у $32,5 \%$ пациентов. У большинства пациентов зафиксировано удовлетворительное качество жизни $(42,1 \%)$ Следует отметить, что у абсолютного большинства $(67,5 \%)$ пациентов качество жизни по DDQ - 15 нельзя признать приемлемым.

Отличные и хорошие показатели качества по ИКЖФХК получено у 74,7\% пациентов в отдаленные сроки после ваготомии с органосохраняющим и органощадящим операциями на желудке. Удовлетворительные результаты получения у $19,2 \%$ пациентов. И у 6,1\% результаты признаны плохими.

У большинства пациентов 46,2\% получены хорошие результаты качества жизни, отличные у $28,8 \%$, удовлетворительные у 21,4\% и лишь у 3,6\% плохие. Для большей объективности полученных отдаленных результатов выполненных операций на желудке, нами они оценены и по классификации Visick (1948) - таблица № 3

Секреторная функция желудка в отдаленном периоде, после резекционных операций.

При изучении отдаленных результатов операций на желудке необходимо оценить прежде всего его секреторную функцию.

В I группе больных (после резекционных желудок операций) в отдаленном периоде секреторная функция желудка изучена у 32 больных, следует отметить, что после резекции желудка, особенно после операции Гофмейстера - Финстерера получить достоверные показатели секреции желудка сложно.

Bo II группе больных (с органосохраняющими операциями с ваготомией) секреторная функция изучена у 40 пациентов.

\section{Результаты и их обсужление}

Непосредственные результаты резекционных методов хирургического лечения стенозирующих язв ДПК сопровождались более частыми осложнениями и наихудшими результатами по сравнению с органосохраняющими операциями с ваготомией.

Летальность после резекционных желудок операций составила 2,3\%, после органосохраняющих с ваготомией летальных случаев не было.

Общее число послеоперационных осложнений (53,3\%) наблюдалось чаще в группе больных перенесших резекцию желудка по сравнению с органосохраняющими операциями (23,1\%). После резекции желудка по Гофмейстеру - Финстеренру и по Бильрот — I наблюдали несостоятельность швов с одним летальным исходом. В этой же группе больных у 5 развилась картина послеоперационного панкреатита, у 4-х средней степени тяжести, купированная медикаментозной терапией, у одного развился инфицированный панкреонекроз с летальным исходом.

Одним из характерных для операций на желудке послеоперационных осложнений является гастростаз. В группе с органосохраняющими операциями и ваготомией гастростаз развился у 4,8\%, после резекционных операций - у 10,4\%.

Из дренирующих желудок операций предпочтение отдавалось гастродуоденостомии по Финнею.

\section{Отдаленные результаты изучены в сроки от 3 до 30 лет после операций}

В I группе из 84 выписанных после резекций желудка в отдаленные сроки удалось обследовать 64 больных. Из них у 43 (67,2\%) выявлены различные патологические состояния (таб.1) Рецидив язвы - 5 (7,8\%), демпинг синдром средней степени - 7 (10,9\%), желу- 
дочный дискомфорт - 14(22,6\%) вегетоастенический синдром - 11 (17\%) и железодефицитная анемия - 4 (9,4\%). В этой группе только у 21 (31,3\%) пациентов не выявлено патологических состояний.

B II группе пациентов, перенесших органосохраняющие и органощадящие операции с ваготомией, обследовано 83 пациента. У 18 (21,6\%) из них (табл. 2) выявлены различные патологические состояния. Рецидив язвы - 2 (2,4\%), демпинг синдром - 2 (2,4\%). Наиболее частым симптомом в этой группе был желудочный дискомфорт - 7 (8,3\%). В этой группе имели одно серьезное осложнение в отдаленном периоде. У одной больной через 2 года после дуоденопластики, СПВ развился стеноз II - III степени анастомоза. При этом у нее не было рецидива язвы. Рубцевание анастомоза шло без язвенного процесса, вследствие технических погрешностей выполнения дуоденопластики. Этой больной выполнен гастродуоденоанастомоз по Финнею с хорошими отдаленными результатами в течение 12 лет.

Сравнение отдаленных результатов после операции Финнея (51) и дуоденопластики (21), по клиническим и рентген - эндоскопическим критериям, преимущество какой либо методики выявлено не было. Единственное, что отмечено, в первые 2-3 года после операции заброс содержимого из ДПК в желудок чаще отмечается после операции по Финнею. Через 3 года разница по результатам этих методик практически нивелируется.

Качество жизни после резекционных операций показано на таблице 3, у абсолютного большинства пациентов оно признанно удовлетворительным и плохим (73\%).

В группе больных с органосохраняющими вмешательствами с СПВ качество жизни в отдаленном периоде у 72,8\% признанно высоким и у 21,4 удовлетворительным-11,6\% и плохим у 3,6\%.

Полученные показатели кислотопродуктивной функции желудка в отдаленном периоде выявили, что у больных с отличными и хорошими отдаленными результатами было нормацидное состояние желудочной секреции, не зависимо от объема операции на желудке.

у больных с удовлетворительными результатами операции отмечено резкое угнетение секреторной функции желудка.

И у больных с плохими результатами операции (рецидив язвы) выявлено повышение, как в базальной фазе, так и в стимулированной фазе секреции.
Таким образом, у больных перенесших различные операции на желудке по поводу язвенной болезни, результаты операции в отдаленном периоде коррелируются с показателями желудочной фазы секреции. У больных с хорошими отдаленными результатами выявлено - нормацидное состояние желудочной секреции. При плохих результатах, с рецидивами язвы, у всех выявлена гиперсекреция желудочного сока.

При этом, лучшие отдаленные результаты получены после органосохраняющих операциях с СПВ.

\section{Выво $\triangle \mathrm{b}$}

Резекционные методы лечения дРяС ДПК сопровождаются большим числом ранних послеоперационных осложнений, отдаленных патологических синдромов, более высоким процентом летальных исходов по сравнению с органосохраняющими, поэтому, у этой категории больных, в функциональном плане эти вмешательства патогенетически менее выгодны и должны выполняться по строгим и индивидуальным показаниям.

Из дренирующих желудок операций при стенозе пилородуоденального канала наиболее предпочтительной является операция Финнея с СПВ.

При постбульбарном стенозе ДПК патогенетически наиболее обоснована дуоденопластика по В.И.Оноприеву с СПВ.

Таким образом, на основании представленного выше материала можно предложить, что при стенозирующей язве пилородуоденального канала органосохраняющие операции с СПВ дают в абсолютном большинстве случаев хорошие непосредственные и отдаленные результаты.

Систематический обзор на основе принципов доказательной медицины, проведенный Бебуришвили А.Г. и соавт. показывает, что рандомизированные клинические исследования (РКИ), имеют определенные изъяны, не позволяющие окончательно регламентировать подходы по лечению пилородуоденального стеноза язвенной этиологии [2]. В рамках доказательной медицины выводы и практические рекомендации по лечению язвенного пилородуоденального стеноза могут быть сформулированы по результатам отдельных РКИ и заключаются в следующем: при выборе способа оперативного лечения необходимо дифференцировать расположение язвенного дефекта, поскольку наиболее часто осложняются стенозированием язвы пилорического канала и препилорические язвы (уровень доказательности 1); чем ниже располагается язвенный дефект, тем 
более эффективными являются селективные варианты ваготомии; при пилорической и препилорической язве рецидив после стволовой ваготомии наблюдается реже (уровеньдоказательности 1); селективные способы ваготомии без дренирующих вмешательств возможны только при «функциональном» стенозе, на фоне обострения язвенной болезни (уровень доказательности 1); при «органическом» стенозе селективные варианты ваготомии возможны (вероятность возникновения постваготомической диареи снижается), но менее целесообразны, так как приходится дренировать желудок (уровень доказательности 1); дренировать желудок после ваготомии возможно как путем пилоропластики, так и с помощью гастроэнтеростомии (уровень доказательности 1); при пилородуоденальном язвенном стенозе пилоропластику лучше делать по Финнею, а не по Гейнике-Микуличу (уровень доказательности 1); ваготомия (стволовая или селективные способы), сочетанная с антрумэктомией, дает меньшую частоту рецидива язвенной болезни, чем ваготомия, дополненная пилоропластикой (уровень доказательности 1); при восстановлении целостности желудочно-кишечного тракта после антрумэктомии предпочтение следует отдавать методике Ру или Бильрот I (уровень доказательности 1)[2].

\section{ЛИТЕРАТУРА}

1. Авакимян В.А. Язвенная болезнь (вопросы патогенеза)// Кубанский научный медицинский вестник.2007.-№ 4-5.С.12-17.

2. Бебуришвили А.Г., Федоров А.В., Сажин В.П., Панин С.И., Д.м.н., проф. Зюбина.-Хирургическое лечение язвенного пилородуоденального стеноза// Хирургия. Журнал имени Н.И. Пирогова 2019, № 4, С. 94-99

3. Бойко В.В., Ткач С.В., Грома В.Г., Шевченко А.Н., Лыхман В.Н., Багиров Н.В. - Оптимизация хирургического лечения пилородуоденальных стенозов язвенной этиологии//Вестник хирургии Казахстана 2013.-№ 2. С. 32-37

4. Горбунов В.Н. Осложненные гигантские пилородуоденальные язвы: клиническая картина, диагностика, хирургическое лечение. Горбунов В.Н., Нагиев Э.Ш., Столярчук Е.В. // Вестник хирургической гастроэнтерологии. — 2009.— № 3 - C. 63-69.

5. Дурлештер В.М., Дидигов М.Т.—- Анализ результатов и сравнительная оценка лечения декомпенсированного рубцово-язвенного стеноза двенадцатиперстной кишки.

6. Кузин Н.М. Селективная проксимальная ваготомия с дуоденопластикой в лечении дуоденальных стенозов у больных язвенной болезнью двенадцатиперстной кишки. Кузин Н.М., Алимов А.Н. // Хирургия.— 1997.— № 4.—C. 38-43.

7. Кузин Н.М. Эффективность хирургического лечения дуоденальной язвы. Кузин Н.М., Крылов Н.Н. // Хирургия. — 1999.— № 1.— С. 17-20.

8. Кульчиев А.А., Морозов А.А., Тедеев С.У., Галачиев С.М., Дзбоев Д.М. Хирургическое лечение рубцово-язвенного тсеноза пилородуоденального канала//Вестник хирургической гастроэнтерологии. 2015. № 3-4. С. 62-70.

9. Лобанков В.М. Хирургия язвенной болезни на рубеже XXI века. // Хирургия. — 2005. — № 1. — C. 58-64.

10. Окоемов М.Н. Сравнительная характеристика эффективности операций при язвенном пилородуоденальном стенозе. Окоемов М.Н., Кузин Н.М., Крылов Н.Н. // Хирургия. — 2002. — № 5. - С. 26-29.

11. Оноприев В.И. Дуоденопластика - новый тип дренирующей операции при селективной проксимальной ваготомии у больных со стенозирующими язвами двенадцатиперстной кишки. Оноприев В.И., Замулин Ю.Г., Сиюхов Ш.Т. // Всероссийская научная практическая конференция хирургов. Тезисы докл. Саратов 1980.- C. 239-240

12. Yamaguchi T., Ichikawa D., Kurioka H. et al. Postoperative clinical evaluation following pyloruspreserving gastrectomy // Hepatogastroenterology. - 2004. Vol. 51, № 57. P. 883-886. 